\title{
Interactions between immune, stress-related hormonal and cardiovascular systems following strenuous physical exercise
}

\author{
D. MENICUCCI 1,2 , A. PIARULLI3,2, F. MASTORCl' , L. SEBASTIANI ${ }^{4}$, M. LAURINO ${ }^{5,2}$, \\ E. GARBELLA ${ }^{1}, 2$, C. CASTAGNINI ${ }^{7}$, S. PELLEGRINI ${ }^{5}$, V. LUBRANO ${ }^{6}$, G. BERNARDI ${ }^{5}$, \\ M.R. METELLI' ${ }^{8}$ R. BEDINI ${ }^{1,2}$, A. L'ABBATE ${ }^{1,2}$, A. PINGITORE ${ }^{1,2}$, A. GEMIGNANI ${ }^{1,2,5}$ \\ ${ }^{1}$ Institute of Clinical Physiology, National Research Council (CNR), Pisa, Italy; ${ }^{2}$ Extreme Centre, \\ Institute of Life Sciences, Scuola Superiore Sant'Anna, Pisa, Italy; ${ }^{3}$ PERCRO, Perceptual Robotics \\ Laboratory, Scuola Superiore Sant'Anna, Pisa, Italy; ${ }^{4}$ Department of Translational Research on \\ New Technologies in Medicine and Surgery, University of Pisa, Pisa, Italy; ${ }^{5}$ Department of Surgery, \\ Medical, Molecular and Critical Area Pathology, University of Pisa, Pisa, Italy; ${ }^{6}$ Tuscany Gabriele \\ Monasterio Foundation, FTGM, Pisa, Italy; ${ }^{7}$ Department of Preclinical and Clinical Pharmacology, \\ University of Florence, Italy; ${ }^{8}$ Laboratory of Specialized Clinical-Chemical Analysis, AUOP, Pisa, Italy
}

\section{A B S T R A C T}

Physical exercise represents a eustress condition that promotes rapid coordinated adjustments in the immune, stress-related hormonal and cardiovascular systems, for maintaining homeostasis in response to increased metabolic demands. Compared to the tight multisystem coordination during exercise, evidence of between-systems cross talk in the early post exercise is still lacking. This study was aimed at identifying possible interactions between multiple systems following strenuous physical exercise (Ironman race) performed by twenty well-trained triathletes. Cardiac hemodynamics, left ventricle systolic and diastolic function and heart rate variability were measured along with plasma concentrations of immune messengers (cytokines and $C$-reactive protein) and stress-related hormones (catecholamines and cortisol) both $24 \mathrm{~h}$ before and within 20 min after the race. Observed changes in antiinflammatory pathways, stress-related hormones and cardiovascular function were in line with previous findings; moreover, correlating parameters' changes (post versus pre-race) highlighted a dependence of cardiovascular function on the post-race biohumoral milieu: in particular, individual post-race variations of heart rate and diastolic function were strongly correlated with individual variations of anti-inflammatory cytokines, while individual baroreflex sensitivity changes were linked to IL-8 increase. Multiple correlations between anti-inflammatory cytokines and catecholamines were also found according with the autonomic regulation of immune function. Observed post-race cytokine and hormone levels were presumptively representative of the increases reached at the effort end while the cardiovascular parameters after the race were measured during the cardiovascular recovery; thus, results suggest that sustained strenuous exercise produced a stereotyped cardiovascular early recovery, whose speed could be conditioned by the immune and stress-related hormonal milieu.

\section{Key words \\ Cardiovascular function •Inflammation $\bullet$ Strenuous exercise}

\section{Introduction}

Physical exercise represents an eustress condition that requires rapid coordinated adjustments in the cardiorespiratory, endocrine and immune systems, which are crucial for the maintenance of homeostasis in response to increased metabolic demands (Martins-Pinge, 2011). 
This implies increasing cardiac output and diverting blood flow to skeletal muscles, heart and brain, as well as by a rapid mobilization of energy stores for supplying the increased muscular activity. To this aim the stroke volume is largely increased by means of various mechanisms involving velocity conduction, myocardial contractility and relaxation sustained by a marked activation of the autonomic nervous system (ANS). Indeed, immediate vagal withdrawal is associated to an initial increment of heart rate (HR), which is followed by the effect of cardiac sympathetic activation that sustains further HR increases. The higher HR during exercise implies also a resetting of the baroreflex function curve to a new operational point, which is characterized by a lower gain as indicated by the markedly reduced baroreflex sensitivity (BRS) (Coote, 2010). These adaptive modifications are controlled by feedbacks from exercising muscles (Coote, 2010) as well as by central commands along with its peripheral components represented by the hypothalamic-pituitary-adrenal axis releasing cortisol and the sympathetic adrenal medullary axis secreting catecholamines (adrenaline [Ad] and noradrenaline [Nad]). Also, physical exercise, particularly in trained individuals, promotes an anti-inflammatory environment, mainly sustained by plasma concentration increases of several cytokines whose level depends on intensity and duration of effort, as well as on muscle mass and physical fitness. This response is different from other stress conditions (i.e. depression or sickness behavior, Maes et al., 2012; Hashmi et al., 2013), in which the classical pro-inflammatory cytokines IL-1beta and TNF-alpha are increased. The anti-inflammatory cytokines milieu characterizing exercise can be promoted by catecholamines, which are known to potentiate IL-10 release (van der Poll et al., 1996), stimulate IL-6 (Steensberg et al., 2001) and inhibit pro-inflammatory cytokines (van der Poll et al., 1996). Moreover, skeletal muscle can be per se a significant source of circulating cytokines and other peptides classified as "myokines" (Pedersen, 2011); in particular IL-6, has been associated to both muscle contraction (Pedersen et al., 2001) and glycogen depletion (Peake et al., 2005) thus acting as an energy sensor for mobilizing extracellular substrates in a hormone-like fashion. Also, IL-6 performs anti-inflammatory effects by inhibiting the production of TNF-alpha and IL-1beta while inducing an increase of IL-1ra and IL-10 (Ostrowski et al., 1999; Petersen and Pedersen, 2005) and contributing to the maintenance of high plasmatic levels of cortisol for several hours after exercise (Streensberg et al., 2003).

Previous studies highlighted that intensity, duration of exercise as well as training and fitness affect the heart rate recovery. In general, the higher is the training level or the better is the fitness the faster is the recovery (Coote, 2010); while the higher is the effort intensity the longer is the recovery duration. In general, the cardiovascular parameters approach baseline levels within minutes or a few hours after the effort end (Murrel et al., 2007). This dynamics is faster compared to the recovery of the biohumoral milieu since blood-circulating factors such as cytokines and stress hormones exhibit $24 \mathrm{~h}$ or longer recovery times (Neubauer et al., 2008).

However, different dynamics do not preclude multisystem interactions; on the contrary the nearly steady-state of the post-exercise biohumoral milieu have facilitated the identification of association with the cardiovascular (CV) function as shown in a recent study of ours on Ironman Triathletes where we found an association between pulmonary edema produced by the inflammatory response and cardiac stroke volume alterations at the end of the competition. (Pingitore et al., 2011).

In light of this, aim of the present study was to investigate the possible influences of altered immune and hormonal milieu produced by a long lasting physical exercise on the CV system studied at the cessation of effort. As sustained exercise model we used an Ironman Triathlon competition, for which it has been shown systemic inflammatory, hormonal and $\mathrm{CV}$ responses in line with the above-mentioned general response (Pingitore et al., 2011; Suzuki et al., 2006).

In the Discussion section, the associations identified between the different systems have been interpreted taking account of the possible ANS modulations.

\section{Materials and methods}

\section{Participants}

Twenty male triathletes were enrolled from participants to an official Ironman Triathlon competition composed by three sport segments: $3.8 \mathrm{Km}$ swim- 
ming, $180 \mathrm{Km}$ cycling and $42.2 \mathrm{Km}$ running, performed consecutively. Subjects were recruited based on age (30 to 45 years), on experience in Ironman races (5 to 10 years), and according to a common weekly training plan that is to perform at least one of the segments composing a complete Ironman Triathlon, nearly each day. All participants had a license from their national triathlon federation for competing, thus we did not perform any preliminary medical evaluation. The Ethics Committee of the University of Pisa approved the study, and all athletes gave their written informed consent.

\section{Experimental protocol}

Both the evening before (12h pre-race) and within 10 minutes after (post-race) the end of the Ironman competition, the athletes underwent blood sampling for hormonal, inflammatory and biochemical assays and CV evaluation. CV study was performed 10 minutes after the pre- and post-race blood sampling and consisted of the echocardiographic study and of 6 minutes Electrocardiogram (ECG) and continuous blood pressure (BP) recordings during resting.

Echocardiographic examination was performed using a trans-thoracic two-dimensional and Doppler echocardiograph (MY LAB 30; Esaote, Florence, Italy) with a broadband (2-4 MHz) phased array transducer. We recorded an apical four-chamber view loop (4s duration) and a pulsed-wave Doppler tracing transmitral blood flow.

ECG was acquired by means of skin electrodes placed according to the modified limb lead II montage, whereas the BP signal was acquired by a photoplethysmograph-based volume-clamp method (Finapres, Ohmeda, Englewood, CO) with a cuff on the middle phalanx of the third finger. Signals were acquired with a sampling rate of $500 \mathrm{~Hz}$.

\section{Analysis of cardiovascular function Echocardiography}

All echocardiographic measures were made offline by the same cardiologists, according to American Society of Echocardiography recommendations (Lange et al., 2005).

For the systolic function, end-diastolic and endsystolic left ventricular volumes (EDV and ESV, respectively) were measured (Folland et al. 1979), left ventricular stroke volume (SV) was calculated as the difference between ESV and EDV; ejection frac- tion (EF) was calculated as 100* (SV/EDV); longitudinal strain was measured with a dedicated software package (XStrain; Esaote) (Di Bella et al., 2010).

For the diastolic function, E-wave, representing the early passive filling of the left ventricle, and E', measuring the early diastolic velocity of the septal and lateral sides of the mitral annulus, were obtained by Pulsed wave Doppler and tissue Doppler imaging, respectively. We used the E/E' ratios in order to obtain pre-load-independent indices of left ventricle diastolic function since it correlates to mean pulmonary capillary wedge pressure and with the left ventricular filling pressure, according with Nagueh formula (Nagueh et al., 1997).

Finally, the systemic vascular resistance (SVR) was estimated as the ratio between mean BP and cardiac output.

\section{ECG and BP signal analysis}

We used ECG time series in order to derive the inter-beat interval series (NN series) by detecting $\mathrm{R}$ wave peaks with a derivative/threshold algorithm (Chiarugi et al., 2007; Laurino et al., 2012). Systolic blood pressure (SBP) series were extracted on the basis of BP signal peak detection within each NN epoch.

From the ECG and BP signals of each condition we derived the mean $\mathrm{hr}$ ( $\mathrm{HR}$, derived from the average of all $\mathrm{NN}$ intervals), the HRV, i.e. the variability of NN series in the low and high frequency bands (LF: 0.04-0.15 Hz and HF:0.15-0.4 Hz) (Task Force, 1996), and the BRS, i.e. how variations of SBP induce variations on HR.

The baroreflex was characterized using the spontaneous sequence method (Bertinieri et al., 1985) analyzing the spontaneous covariation of SBP and NN sequences in the time domain. The BRS was estimated by searching for sequences of at least three consecutive cardiac cycles in which both $\mathrm{NN}$ and SBP monotonically increased or decreased simultaneously, respectively by at least $2 \mathrm{~ms}$ per beat and $1 \mathrm{mmHg}$ per beat. For each sequence, the slope of regression line between SBP and the paired NN values represented the instantaneous BRS (units: $\mathrm{ms} / \mathrm{mmHg}$ ). We considered reliable only those estimates derived from sequences having $\mathrm{r}^{2}$ greater than 0.5. The BRS index related to each condition was provided as the average value of the retained instantaneous BRS indexes. 


\section{Analysis of blood markers}

Plasma concentrations of cytokines (IL-1beta, IL-1 ra，IL-5，IL-6，IL-8，IL-10，IL-12， MCP-1 and TNF-alpha) and C-reactive protein (CRP) were determined quantitatively by ELISA (Bender Medsystems, Austria, R\&D Systems, Italy and Invitrogen, USA, Diagnostics Biochem Canada Inc, Canada). Serum cortisol was measured with a fluorescent polarization immunoassay method with the TDx system (Abbott Diagnostics, USA). For the measurement of plasma catecholamines, automated HPLC analyzer was used (HCL-725 CA, Tosoh Corporation, Japan). Plasma concentrations of electrolytes were measured by ion selective electrodes (ISE) technique (Beckman Coulter Synchron CX9PRO), and plasma osmolality derived from the respective concentrations of sodium, urea and glucose.

\section{Statistical analysis}

We studied individual post-race versus pre-race feature changes by means of two-tails paired t-tests on both the blood-derived and the CV-related variables. For each condition and for each variable, tables report group mean $\pm 95 \%$ confidence interval $(95 \% \mathrm{CI})$. Besides, we performed the analysis of correlations between feature changes resting on the hypothesis that the process of adaptation to stress load involves the regulation of systems by the action of coordinated controls. We calculated the Pearson correlations over the subjects between the features changes. For each pair of features, the correlation was considered significant or highly significant according to two thresholds: $|r|>0.60(\mathrm{p}<0.01)$ and to $|\mathrm{r}|>0.48(\mathrm{p}$ $<0.05$ ). The resulting list of significant correlations was organized in a graph.

\section{Results}

All participants completed the competition in a time from 11 to 14 hours; however, one out of twenty was excluded from the study because of body hypothermia $\left(35^{\circ} \mathrm{C}\right)$ and hypotension $(80 / 50 \mathrm{mmHg})$ at the end of the race. The remaining athletes underwent the second CV study within 20 minutes from the finishing-line crossing. Two of them had a very low quality BP signal likely due to peripheral vasoconstriction, which prevented a reliable estimate of BRS. In summary, we had 17 subjects with complete pre- and post-race data. Post-race plasma osmolality (PO), calcium and potassium concentrations were significantly increased, whereas chloride plasma levels were significantly lower compared to the evening before the competition (Table I). These changes were highly coherent across subjects and only sodium did not change significantly in response to exercise. In addition, hematocrit and hemoglobin, assessed in a subgroup of eight athletes, changed only slightly (from $-1 \%$ to $4 \%$ and from -0.3 to 1.3 $\mathrm{g} / \mathrm{dl}$ respectively, both variations being not statistically significant). Thus, the variations of plasma volume related to the race did not significantly affect the actual changes of inflammatory and hormonal parameters.

\section{Cytokines and hormones}

Changes of cytokines and hormones blood concentration from pre- to post-race are shown in Table II and Table III. Post-race plasma concentrations of IL1-ra, IL-6, IL-8, IL-10, MCP-1, CRP and NAd were significantly increased compared to pre-race condition in all subjects; similarly IL-12, Ad and cortisol increased in 15 out of 17 subjects, with small opposite changes for the other subjects. At

\begin{tabular}{|c|c|c|c|c|}
\hline Variable & Pre-race & Post-race & $\Delta$ & p-value \\
\hline Calcium (mg/dL) & $9.1 \pm 0.2$ & $9.7 \pm 0.2$ & $0.7 \pm 0.3$ & $p<0.001$ \\
\hline Potassium (mmol/L) & $4.1 \pm 0.1$ & $4.6 \pm 0.3$ & $0.50 \pm 0.3$ & $\mathrm{p}<0.001$ \\
\hline Chloride (mmol/L) & $106 \pm 1$ & $104 \pm 1.2$ & $-2.0 \pm 2.0$ & $p<0.01$ \\
\hline Sodium (mmol/L) & $138 \pm 1$ & $140 \pm 1$ & $1.0 \pm 1.0$ & ns \\
\hline $\mathrm{PO}(\mathrm{mOsm} / \mathrm{Kg})$ & $277 \pm 2$ & $284 \pm 2$ & $7 \pm 3$ & $p<0.001$ \\
\hline
\end{tabular}




\begin{tabular}{|c|c|c|c|c|}
\hline \multicolumn{5}{|c|}{ Table II. - Plasma values of cytokines and CRP. } \\
\hline Variable & Pre-race & Post-race & $\Delta$ & p-value \\
\hline $\mathrm{IL}-1 \beta(\mathrm{pg} / \mathrm{mL})$ & $0.80 \pm 0.3$ & $0.70 \pm 0.2$ & $-0.13 \pm 0.3$ & $\mathrm{~ns}$ \\
\hline IL-1 ra (pg/mL) & $70.00 \pm 15$ & $340 \pm 80$ & $270 \pm 80$ & $\mathrm{p}<0.001$ \\
\hline $\mathrm{IL}-5(\mathrm{pg} / \mathrm{mL})$ & $20 \pm 3$ & $20 \pm 1.30$ & $0.40 \pm 1.6$ & $\mathrm{~ns}$ \\
\hline $\mathrm{IL}-6(\mathrm{pg} / \mathrm{mL})$ & $0.07 \pm 0.09$ & $16 \pm 5$ & $16 \pm 5$ & $\mathrm{p}<0.001$ \\
\hline $\mathrm{IL}-8(\mathrm{pg} / \mathrm{mL})$ & $0.33 \pm 0.4$ & $4 \pm 3$ & $4.00 \pm 3$ & $p<0.001$ \\
\hline $\mathrm{IL}-10(\mathrm{pg} / \mathrm{mL})$ & $1.90 \pm 1.6$ & $15 \pm 5$ & $13 \pm 5$ & $p<0.001$ \\
\hline $\mathrm{IL}-12(\mathrm{pg} / \mathrm{mL})$ & $200 \pm 10$ & $300 \pm 50$ & $97.10 \pm 49$ & $\mathrm{p}<0.001$ \\
\hline MCP-1 (pg/mL) & $200 \pm 10$ & $580 \pm 96$ & $382.11 \pm 97.63$ & $\mathrm{p}<0.001$ \\
\hline TNF- $\alpha(\mathrm{pg} / \mathrm{mL})$ & $0.30 \pm 0.2$ & $0.2 \pm 0.10$ & $-0.11 \pm 0.27$ & $\mathrm{~ns}$ \\
\hline CRP (mg/dL) & $0.10 \pm 0.05$ & $0.50 \pm 0.3$ & $0.50 \pm 0.3$ & $p<0.001$ \\
\hline
\end{tabular}

\begin{tabular}{|c|c|c|c|c|}
\hline Variable & Pre-race & Post-race & $\Delta$ & p-value \\
\hline Cort (ng/ml) & $83 \pm 19$ & $289 \pm 60$ & $198 \pm 72$ & $p<0.001$ \\
\hline Ad $(\mathrm{pg} / \mathrm{ml})$ & $20 \pm 13$ & $63 \pm 26$ & $43 \pm 24$ & $\mathrm{p}<0.001$ \\
\hline NAd (pg/ml) & $170 \pm 70$ & $743 \pm 134$ & $573 \pm 112$ & $p<0.001$ \\
\hline
\end{tabular}

variance, IL-1beta, IL-5 and TNF-alpha had a scattered behavior with small post-race changes compared to the pre-race levels.

\section{Cardiovascular features}

Parameters derived from ECG and BP recordings showed coherent changes over the subjects: in particular, the mean HR increased and the SBP decreased significantly in the post-race as compared to the prerace condition in all subjects (Table IV). Moreover, the power associated with LF and HF components of the HRV significantly decreased in the post-race, with the exception of three subjects showing slight opposite changes. On average, LF power decrements were smaller than HF ones: hence the LF/HF ratio increased in the post-race. The baroreflex function exhibited a consistent decrease from pre-race to postrace as indicated by the sensitivity index BRS.

Also parameters derived from echocardiography showed coherent changes over the subjects: in particular, the mean stroke volume (SV) significantly decreased in the post-race in all subjects (Table IV). Besides, the other systolic function measures - ejection fraction and longitudinal strain - significantly decreased in the post-race with the exception of two subjects showing slight opposite changes. For the diastolic function neither the septal nor the lateral E/E' ratios were significantly changed despite $2 / 3$ of the subjects showed decreased post-race ratios. Also, the SVR were not significantly changed.

\section{Profiling multisystem responses to the race}

The identification of multisystem stress response profiles was based on correlation analysis that took advantage of the inter-subject variability. Indeed, Fig. 1 summarizes qualitative and numerical results from the correlations between post/pre-race feature changes. The graph shows a cluster tightened by positive high correlations (from 0.69 to 0.97 ) between cytokines IL-1ra, IL-6, IL-8 and IL-12 and MCP-1. This group identifies the anti-inflammatory response core whose individual variability is associated with the magnitude of catecholamines (Ad and NAd) changes, as well as with CRP variations. Besides, cortisol changes were also inversely correlated with CRP changes.

Post versus pre-race differences of the CV features were not intercorrelated (except for the link between 


\begin{tabular}{|c|c|c|c|c|}
\hline Variable & Pre-race & Post-race & $\Delta$ & p-value \\
\hline \multicolumn{5}{|l|}{ Cardiac hemodynamic } \\
\hline $\mathrm{SBP}(\mathrm{mmHg})$ & $126 \pm 12$ & $107 \pm 11$ & $-19 \pm 9$ & $p<0.001$ \\
\hline $\mathrm{DBP}(\mathrm{mmHg})$ & $74 \pm 11$ & $70 \pm 12$ & $-2 \pm 9$ & ns \\
\hline HR (beats/min) & $56 \pm 9$ & $70 \pm 8$ & $15 \pm 8$ & $p<0.001$ \\
\hline SVR (mmHg $\mathrm{min} / \mathrm{ml})$ & $0.02 \pm 0.004$ & $0.01 \pm 0.004$ & $-0.002 \pm 0.004$ & $\mathrm{~ns}$ \\
\hline BRS (ms/mmHg) & $9 \pm 4$ & $5.1 \pm 2.5$ & $-4 \pm 4$ & $p<0.05$ \\
\hline \multicolumn{5}{|l|}{ Heart Rate Variability } \\
\hline $\mathrm{HF}\left(\mathrm{ms}^{2}\right)$ & $700 \pm 400$ & $160 \pm 90$ & $-550 \pm 400$ & $p<0.01$ \\
\hline $\mathrm{LF}\left(\mathrm{ms}^{2}\right)$ & $1600 \pm 500$ & $750 \pm 340$ & $-860 \pm 600$ & $p<0.01$ \\
\hline \multicolumn{5}{|l|}{ LV diastolic function } \\
\hline Septal E/E' & $6 \pm 1$ & $5 \pm 1$ & $-0.6 \pm 1$ & $\mathrm{~ns}$ \\
\hline Lateral E/E' & $4.4 \pm 0.7$ & $4 \pm 1$ & $-0.3 \pm 1$ & ns \\
\hline \multicolumn{5}{|l|}{ LV systolic function } \\
\hline Ejection fraction (\%) & $72 \pm 6$ & $65 \pm 7.0$ & $-6 \pm 5.5$ & $p<0.001$ \\
\hline Longitudinal strain (\%) & $18 \pm 3$ & $16.0 \pm 2.6$ & $-2 \pm 2.70$ & $p<0.05$ \\
\hline Stroke volume $(\mathrm{ml})$ & $119 \pm 24$ & $90 \pm 17$ & $-27 \pm 16$ & $p<0.001$ \\
\hline
\end{tabular}

HR and lateral E/E') but rather they were linked to the changes of different inflammatory markers. In particular, HR and lateral E/E' variations correlated with each marker belonging to the anti-inflammatory core response (IL-6, IL-1ra, MCP-1 and IL-12), besides septal and lateral E/E' were also correlated with NAd, whereas BRS changes were directly correlated with IL-8 changes.

For the systolic function parameters, only the EF changes were connected to the graph as it was significantly correlated to Ad changes. For the HRV features, HF and LF post/pre-race differences showed a negative correlation with SV changes and a positive one with SVR changes.

\section{Discussion}

Taking advantage of a regular Ironman competition, we selected a group of well-trained athletes and we studied them both $12 \mathrm{~h}$ before the race, and at the beginning of the post-race recovery. The selection of subjects and their involvement in the same race has allowed us to set both exercise intensity and training level. The joint immune, stress-related hormonal and $\mathrm{CV}$ characterization has allowed a multisystem assessment of the response to sustained strenuous exercise. Our data exhibit new links between response magnitudes of different systems: we connected the CV early recovery with the level of circulating catecholamines and cytokines measured at the effort end. As discussed in the following, for example, subjects showing higher post race HR (compared to pre-race levels) were also the high responders for the inflammatory response.

\section{Hormonal modulation of inflammation}

The observed pattern of cytokines activation after strenuous exercise indicated that endurance exercise could generate local muscle inflammatory reactions possibly related to muscle degenerative events. In fact, CRP, MCP-1and IL-8 levels that have a key role in local inflammation are significantly increased. This is in line with a previous study in Ironman athletes showing that slow recovery of CRP values (5 days) was associated to incomplete muscle recovery (Neubauer et al., 2008). However, the amplitude of local reaction is not associated to a corresponding systemic inflammatory response. Indeed, increases in IL-1beta and TNF-alpha were not significant and plasma levels of anti-inflammatory cytokines, IL-6, IL-1ra and IL-10 were higher 


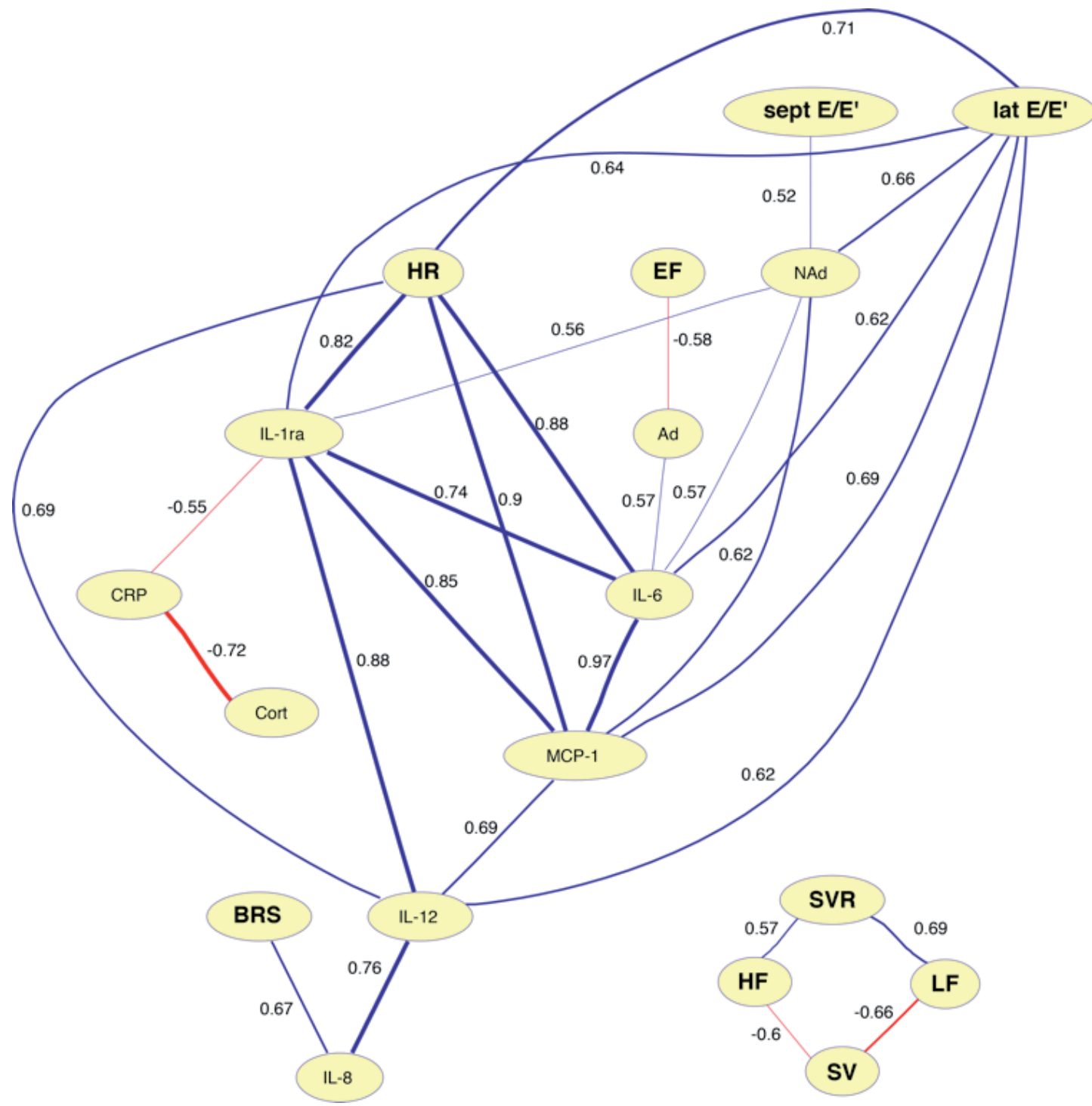

Fig. 1. - The identification of multisystem stress response profiles based on correlations between post/pre-race feature changes. The lines between features indicate the significant links; the color of line provides indication of direct (black) or inverse (red) proportionality, whereas thickness increases with correlation strength and the numerical value is the related correlation coefficient.

than pre-race values (Pingitore et al., 2011). The dissociation between local and systemic reaction has been previously described (Marklund et al., 2013). The pattern of anti-inflammatory cytokine, likely released by contracting muscles itself could then contribute to prevent inflammation overshooting, thus attenuating the systemic immune responses through the balance between anti and pro-inflammatory pathways (Fehrenbach and Schneider, 2006). Actually, we found a negative correlation between IL-1ra and CRP, i.e. subjects with the highest antiinflammatory response, as indicated by their IL-1ra increase, showed the lowest CRP increases. Besides, we identified a negative link between CRP and cortisol, a classical marker of the stress response and a key neuroendocrine modulator of the immune system during exercise (anti-inflammatory role) (Papanicolaou et al., 1996).

As concerns IL-6, its post-race plasma concentration is more than 100 -fold higher with respect to pre-race value and, as demonstrated by previous findings, it takes at least one day to return to the pre-race level (Jeukendrup et al., 2000; Suzuki et al., 2006). Since IL-6 is a sensor of energy stores and promotes 
substrates mobilization, its slow recovery suggests that, besides the anti-inflammatory effect, IL-6 high levels could either indicate an impairment in muscle glycogen restoration or an efficient mechanism of high glucose up-take after exercise. As far as the catecholamines are concerned, we observed increases of both adrenaline and noradrenaline; these increments were correlated with cytokine increments, likely due to several reasons: 1) adrenaline-induced decrease in IL-6 plasma clearance (Steensberg et al., 2001); 2) adrenaline-induced potentiation of IL-6 synthesis in skeletal muscles (Frost et al., 2004); 3) direct effect, via noradrenaline receptors, on macrophages production of cytokines (Pavlov and Tracey, 2004). In addition, the positive correlation between noradrenaline and IL-1ra/MCP-1 could be secondary to the correlations with the IL-6.

A specific role in promoting post-race restoration can also be ascribed to MCP-1; in fact it has been suggested that in healthy skeletal muscle following acute direct or contraction-induced injuries, MCP-1 could promote muscle regeneration and functional recovery through regulation of monocyte/macrofage recruitment or activation (Tidball, 2005; Warren et al., 2005). The strong correlation between MCP-1 and IL- 6 could thus be suggestive of a coordinated activation of mechanisms involved in both structural and metabolic post exercise recovery.

\section{Immune and hormonal modulation of post-race cardiovascular function}

Post-race cytokine and hormone levels are presumptively representative of the increases reached at the effort end while $\mathrm{CV}$ parameters have a faster dynamics and their post-race values are indicative of recovery speed (Tulppo et al., 2011).

The CV parameters measured immediately after the end of the race revealed a pattern of changes in line with previous literature on Ironmen (Gratze et al., 2005; Niemela et al., 2008); specifically, sympathetic activity, as indicated by the marked decrease in BRS and the increase in HR, is still higher than pre-race values while post-race SBP was lower with respect to the pre-race level. Besides, post-race LV systolic function, as indicated by EF, SV and LS, was decreased consistent with previous findings about beta-adrenergic receptors desensitization following physical strenuous exercise. Indeed, experiments with the administration of sympathomimetic drugs such as dobutamine or isoproterenol highlighted that the dose-response curve of changes in HR or LV contractility shifts to the right as a function of exercise duration/intensity (Eysmann et al., 1996). At variance, the post-race LV diastolic function, as indicated by the E/E' ratios, was unaltered (Ha et al., 2003).

In general, the post-exercise $\mathrm{CV}$ recovery is characterized by a rapid return to basal values due to a cardiac parasympathetic reactivation immediately after the cessation of physical activity, associated with a gradual subsiding of sympatho-adrenal action. This sympathetic-parasympathetic interaction, which is centrally mediated but also regulated by muscle afferents (i.e. metaboreceptors) and circulating catecholamines, is strongly influenced by duration and type of exercise with longer time courses after endurance races to allow sufficient organ perfusion and to prevent circulatory collapse (Coote, 2010). Besides, the low level of blood pressure is likely due to the sustained post-exercise vasodilation that promotes high glucose availability to skeletal muscles allowing the metabolic restoration, and that is mainly induced by exercise-related local vasodilator mechanisms (i.e. histamine receptors activation and nitric oxide, Clifford and Hellsten, 2004; Halliwill et al., 2012) as well as by a blunted transduction of sympathetic output into vasoconstriction (i. e. presynaptic inhibition of NAd release or increased reuptake of NAd, Halliwill et al., 2012).

Up to now, no direct relations between CV changes, inflammatory and hormonal patterns after sustained exercise have been described. The most interesting result of the study is the striking association between the individual early recovery of $\mathrm{HR}$ and diastolic function $(\mathrm{E} / \mathrm{E}$ ') and the post-exercise inflammatory changes (IL-6, IL-12, IL-1ra, MCP-1), with the highest positive correlation with the inflammatory cytokine MCP-1. This finding indicated that individuals with the slowest post-race $\mathrm{CV}$ recovery (e.g. high HR changes) have the highest inflammatory cytokines levels.

To date, a direct link between CV function and cytokines during strenuous exercise remains undetermined. The link between HR and E/E' as representative of preload (Nagueh et al., 1997) suggests interpreting the correlations with the cytokine pattern in a unitary framework. In particular, the stronger correlations of HR-cytokines compared to lateral 
E/E'-cytokines could indicate that these latter relations could derive from the HR-E/E' one. Actually, it has been previously demonstrated (Tracey, 2002) that the autonomic nervous system can modulate the anti-inflammatory response in sepsis condition. In addition, pro-inflammatory cytokines can interact with the brain both directly and via vagal afferents activation, exerting regulatory effects on the autonomic nervous system activity. For example, inflammatory cytokines released in response to LPS administration have been demonstrated to increase HR (Fairchild et al., 2009). Thus, post-race high levels of cytokines could buffer the recovery of HR by enhancing sympathetic activity.

The correlation between EF changes and adrenaline post-race level could be explained by the phenomenon adrenergic receptors desensitization with exercise; in fact when testing sympathetic reactivity by means of isoproterenol, the increase in amount of isoproterenol needed to obtain a determined HR effect after prolonged exercise was closely related to the decrease in ejection fraction (Eysmann et al., 1996). Previous studies have also shown that adrenergic receptors desensitization provide a contribution to the decrease of systolic function (Hart et al., 2006; Banks et al., 2010).

The positive relationship between BRS and IL-8, that plays a key role in local inflammation (Harada et al., 1996), suggests its contribution to the baroreflex function inhibition. The exact mechanism responsible for post-race baroreflex resetting is still unknown, however research over the last decade focused on the role of input from muscle afferents to the NTS as a key modulator of the sympathetic output from the CV control centers (Halliwill et al., 2013). Since inflammatory cytokines are known to modulate NTS activity via vagal afferents activation we can assume their role in the delayed resetting of the reflex (Watkins et al., 1995). Moreover, despite the lack of any correlation between IL-6 and BRS, recent studies in the animal model suggested a possible role of IL-6 within the NTS in the attenuation of the baroreceptor reflex (Takagishi et al., 2010). Finally, the lack of significant correlation between HR changes and sympathovagal balance is in line with previous studies in which HRV failed to reflect changes in autonomic modulation during exhaustive exercise in trained subjects (Pichon et al., 2004). Indeed, the observed LF reduction, in spite of the sympathetic activation, is the consequence of $\mathrm{CV}$ system stabilization due to peripheral resistances decrease. Experimental evidence and computational simulations have shown that oscillations in HR and arterial pressure are markedly attenuated when peripheral resistances are decreased as during exercise (Ottesen et al., 1997). As a confirmation, we found significant correlations between post-race decreases in SVR and depression in both LF and HF bands of HRV.

\section{Conclusion}

Peculiarity of the present paper was the simultaneous assessment of inflammatory, stress-related hormonal and cardiovascular function pre and post Ironman Triathlon race. The present work discloses a window on the CV function after physical exercise resulting in a complex integration with stress hormones and inflammatory responses. This suggests further work for a long-term follow-up of the multisystem integration during the post exercise recovery in order to obtain new information of recovery efficiency.

\section{Acknowledgements}

We thank the Ironmen athletes who participated into the study as well as the "Parco Nazionale dell'Asinara" for logistics.

\section{References}

Banks L., Sasson Z., Busato M., Goodman J.M. Impaired left and right ventricular function following prolonged exercise in young athletes: influence of exercise intensity and responses to dobutamine stress. Appl. Physiol., 108: 112-119, 2010.

Bertinieri G., di Rienzo M., Cavallazzi A., Ferrari A.U., Pedotti A., Mancia G. A new approach to analysis of the arterial baroreflex. J. Hypertens., 3 : S79-81, 1985.

Chiarugi F., Sakkalis V., Emmanouilidou D., Krontiris T., Varanini M., Tollis I. Adaptive Threshold QRS Detector with Best Channel Selection Based on a Noise Rating System. Computers in Cardiology, 34: 157-160, 2007.

Clifford P.S. and Hellsten Y. Vasodilatory mechanisms in contracting skeletal muscle. J. Appl. Physiol., 97: 393-403, 2004. 
Coote J.H. Recovery of heart rate following intense dynamic exercise. Exp. Physiol., 95: 431-440, 2010.

Di Bella G., Gaeta M., Pingitore Oreto G., Zito C., Minutoli F., Anfuso C., Dattilo G., Lamari A., Coglitore S., Carerj S. Myocardial deformation in acute myocardatis with normal left ventricular wall motion: a cardiac magnetic resonance and 2-dimensional strain echocardiographic study. Circ. J., 74: 1205-1213, 2010.

Eysmann S.B., Gervino E., Vatner D.E., Katz S.E., Decker L., Douglas P.S. Prolonged exercise alters beta-adrenergic responsiveness in healthy sedentary humans. J. Appl. Physiol., 80: 616-622, 1996.

Fairchild K.D., Saucerman J.J., Raynor L.L., Sivak J.A., Xiao Y., Lake D.E., Moorman J.R. Endotoxin depresses heart rate variability in mice: cytokine and steroid effects. Am. J. Physiol. Regul. Integr. Comp. Physiol., 97: 1019-1027, 2009.

Fehrenbach E. and Schneider M.E. Trauma-induced systemic inflammatory response versus exerciseinduced immunomodulatory effects. Sports Med., 36: 373-384, 2006.

Folland E.D., Parisi A.F., Moynihan P.F., Ray Jones D., Feldman C.L., Tow D.E. Assessment of left ventricular ejection fraction and volumes by realtime, two-dimensional echocardiography. A comparison of cineangiographic and radionuclide techniques. Circulation, 60: 760-766, 1979.

Frost R.A., Nystrom G.J., Lang C.H. Epinephrine stimulates IL-6 expression in skeletal muscle and C2C12 myoblasts: role of c-Jun NH2-terminal kinase and histone deacetylase activity. Am. J. Physiol. Endocrinol. Metab., 286: 809-817, 2004.

Gratze G., Rudnicki R., Urban W., Mayer H., Schlögl A., Skrabal F. Hemodynamic and autonomic changes induced by Ironman: prediction of competition time by blood pressure variability. J. Appl. Physiol., 99: 1728-1735, 2005.

Ha J.W., Lulic F., Bailey K.R., Pellikka P.A., Seward J.B., Tajik A.J., Oh J.K. Effects of treadmill exercise on mitral inflow and annular velocities in healthy adults. Am. J. Cardiol., 91: 114-115, 2003.

Halliwill J.R., Buck T.M., Lacewell A.N., Romero S.A. Postexercise hypotension and sustained postexercise vasodilatation: what happens after we exercise? Exp. Physiol., 98: 7-18, 2013.

Harada A., Mukaida N., Matsushima K. Interleukin 8 as a novel target for intervention therapy in acute inflammatory diseases. Mol. Med. Today, 2: 482489, 1996.

Hart E., Dawson E., Rasmussen P., George K., Secher N.H., Whyte G., Shave R. Beta-adrenergic recep- tor desensitization in man: insight into post-exercise attenuation of cardiac function. J. Physiol., 577: 717-725, 2006.

Hashmi A.M., Butt Z., Umair M. Is depression an inflammatory condition? A review of available evidence. J. Pak. Med. Assoc., 63: 899-906, 2013.

Jeukendrup A.E., Vet-Joop K., Sturk A., Stegen J.H., Senden J., Saris W.H., Wagenmakers A.J. Relationship between gastro-intestinal complaints and endotoxaemia, cytokine release and the acutephase reaction during and after a long-distance triathlon in highly trained men. Clin. Sci. (Lond), 98: 47-55, 2000.

Lang R.M., Bierig M., Devereux R.B., Flachskampf F.A., Foster E., Pellikka P.A., Picard M.H., Roman M.J., Seward J., Shanewise J.S., Solomon S.D., Spencer K.T., St John Sutton M., Stewart W.J. Recommendations for chamber quantification. J. Am. Soc. Echocardiogr., 18: 1440-1463, 2005.

Laurino M., Menicucci D., Mastorci F., Allegrini P., Piarulli A., Scilingo E.P., Bedini R., Pingitore A., Passera M., L'Abbate A., Gemignani A. Mindbody relationships in elite apnea divers during breath holding: a study of autonomic responses to acute hypoxemia. Front. in Neuroeng., 5: 4, 2012.

Maes M., Berk M., Goehler L., Song C., Anderson G., Gałecki P., Leonard B. Depression and sickness behavior are Janus-faced responses to shared inflammatory pathways. BMC Med., 10: 66, 2012.

Marklund P., Mattsson C.M., Wåhlin-Larsson B., Ponsot E., Lindvall B., Lindvall L., Ekblom B., Kadi F. Extensive inflammatory cell infiltration in human skeletal muscle in response to an ultraendurance exercise bout in experienced athletes. J. Appl. Physiol., 114: 66-72, 2013.

Martins-Pinge M.C. Cardiovascular and autonomic modulation by the central nervous system after aerobic exercise training. Braz. J. Med. Biol. Res., 44: 848-854, 2011.

Murrell C., Wilson L., Cotter J.D., Lucas S., Ogoh S., George K., Ainslie P.N. Alterations in autonomic function and cerebral hemodynamics to orthostatic challenge following a mountain marathon. J. Appl. Physiol., 103: 88-96, 2007.

Nagueh S.F., Middleton K.J., Kopelen H.A., Zoghbi W.A., Quinones M.A. Doppler tissue imaging: a noninvasive technique for evaluation of left ventricular relaxation and estimation of filling pressures. J. Am. Coll. Cardiol., 30: 1527-1533, 1997.

Neubauer O., König D., Wagner K.H. Recovery after an Ironman triathlon: sustained inflamma- 
tory responses and muscular stress. Eur. J. Appl. Physiol., 104: 417-426, 2008.

Niemelä T.H., Kiviniemi A.M., Hautala A.J., Salmi J.A., Linnamo V., Tulppo M.P. Recovery pattern of baroreflex sensitivity after exercise. Med. Sci. Sports Exerc., 40: 864-870, 2008.

Ostrowski K., Rohde T., Asp S., Schjerling P., Pedersen B.K. Pro- and anti-inflammatory cytokine balance in strenuous exercise in humans. $J$. Physiol., 515: 287-291, 1999.

Ottesen J.T. Modelling of the baroreflex-feedback mechanism with time-delay. Math. Biol., 36: 41-63, 1997.

Papanicolaou D.A., Petrides J.S., Tsigos C., Bina S., Kalogeras K.T., Wilder R., Gold P.W., Deuster P.A., Chrousos G.P. Exercise stimulates interleukin-6 secretion: inhibition by glucocorticoids and correlation with catecholamines. Am. J. Physiol., 271: E601-605, 1996.

Pavlov V.A. and Tracey K.J. Neural regulators of innate immune responses and inflammation. Cell. Mol. Life Sci., 61: 2322-2331, 2004.

Peake J.M., Suzuki K., Wilson G., Hordern M., Nosaka K., Mackinnon L., Coombes J.S. Exerciseinduced muscle damage, plasma cytokines, and markers of neutrophil activation. Med. Sci. Sports Exerc., 37: 737-745, 2005.

Pedersen B.K. Muscles and their myokines. J. Exp. Biol., 214: 337-346, 2011.

Pedersen B.K., Steensberg A., Fischer C., Keller C., Ostrowski K., Schjerling P. Exercise and cytokines with particular focus on muscle-derived IL-6. Exerc. Immunol. Rev., 7: 8-31, 2001.

Petersen A.M. and Pedersen B.K. The anti-inflammatory effect of exercise. J. Appl. Physiol., 98: 1154-1162, 2005.

Pingitore A., Garbella E., Piaggi P., Menicucci D., Frassi F., Lionetti V., Piarulli A., Catapano G., Lubrano V., Passera M., Di Bella G., Castagnini C., Pellegrini S., Metelli M.R., Bedini R., Gemignani A., L'Abbate A. Early subclinical increase in pulmonary water content in athletes performing sustained heavy exercise at sea level: ultrasound lung comet-tail evidence. Am. J. Physiol. Heart Circ. Physiol., 301: H2161-2167, 2011.

Steensberg A., Fischer C.P., Keller C., Møller K., Pedersen B.K. IL-6 enhances plasma IL-1ra,
IL-10, and cortisol in humans. Am. J. Physiol. Endocrinol. Metab., 285: E433-E437, 2003.

Steensberg A., Toft A.D., Schjerling P., HalkjaerKristensen J., Pedersen B.K. Plasma interleukin-6 during strenuous exercise: role of epinephrine. Am. J. Physiol. Cell. Physiol., 281: C1001-C1004, 2001.

Suzuki K., Peake J., Nosaka K., Okutsu M., Abbiss C.R., Surriano R., Bishop D., Quod M.J., Lee H., Martin D.T., Laursen P.B. Changes in markers of muscle damage, inflammation and HSP70 after an Ironman Triathlon race. Eur. J. Appl. Physiol., 98: 525-534, 2006.

Takagishi M., Waki H., Bhuiyan M.E., Gouraud S.S., Kohsaka A., Cui H., Yamazaki T., Paton J.F., Maeda M. IL-6 microinjected in the nucleus tractus solitarii attenuates cardiac baroreceptor reflex function in rats. Am. J. Physiol. Regul. Integr. Comp. Physiol., 29: 183-189, 2010.

Task Force of the European Society of Cardiology and the North American Society of Pacing and Electrophysiology. Heart rate variability-standards of measurement, physiological interpretation, and clinical use. Circulation, 93: 1043-1065, 1996.

Tidball J.G. Inflammatory processes in muscle injury and repair. Am. J. Physiol. Regul. Integr. Comp. Physiol., 288: 345-353, 2005.

Tracey K.J. The inflammatory reflex. Nature, 420: 853-859, 2002.

Tulppo M.P., Kiviniemi A.M., Hautala A.J., Kallio M., Seppänen T., Tiinanen S., Mäkikallio T.H., Huikuri H.V. Sympatho-vagal interaction in the recovery phase of exercise. Clin. Physiol. Funct. Imaging, 31: 272-281, 2011.

van der Poll T., Coyle S.M., Moldawer L.L., Lowry S.F. Changes in endotoxin-induced cytokine production by whole blood after in vivo exposure of normal humans to endotoxin. J. Infect. Dis., 174: 1356-1360, 1996.

Warren G.L., Hulderman T., Mishra D., Gao X., Millecchia L., O'Farrell L., Kuziel W.A., Simeonova P.P. Chemokine receptor CCR2 involvement in skeletal muscle regeneration. FASEB J. 19: 413-415, 2005.

Watkins L.R., Maier S.F., Goehler L.E. Cytokineto-brain communication: a review and analysis of alternative mechanisms. Life Sci., 57: 1011-1026, 1995. 